\title{
Review of: "Prestenting and its role in the URS: a study on the advantages it could have in intracorporeal lithotripsy interventions"
}

\author{
Stefania Ferretti ${ }^{1}$ \\ 1 University Hospital of Parma
}

Potential competing interests: The author(s) declared that no potential competing interests exist.

Dear Editor, the study-concept is interesting; we know very well the limits of a retrospective observational study. I would like to suggest the authors review some parts of the paper before editing: 1. Methods: How many experienced surgeons were involved in the procedures? (The results may be affected..); what do you mean with " endoscopic control or radioscopic"? for attesting SFR. You decided intraoperatively? It's necessary to improve this part (which calibre of UAS? Which follow-up for SFR : US/X-ray abdomen vs CTscan at 1 or 3 month? when started the operating time OT?..); Ethical Approval was unmentioned. I suppose theTAB.1 \& 2 belong to M\&M but tehy are not inserted. TAb.1 : Description of population. What do you mean with "previous endoscopic surgery"? There's a very incidence in prestenting group (93,6\%) compared to not prestenting group (17\%). Do you consider the stent insertion in Urgency (I suppose for all 84 pts..more or less..or not? Maybe I didn't understand correctly ) also in this statement? 2. Results: It's necessary to improve the tables with more results 3. Discussion: Not present. How to discuss and motivated the results? at least some hypotheses need to be formulated. Generally for large stones or multiple stones (RIRS) the possibility to insert a more generous UAS reduces the hyperpressure risks (etc.) and increases the stone fragments removal with an increase in SFR. Prestented patients shoud be more fit. Have you ever had failures to put the same UAS in non-prestented 180 patients? 4. Conclusions had to underline pro and contra of results 5. references: the citated papers are only in the introduction. Section to improve in discussion- 